\title{
HP-SFC Algorithm for MmWave Massive MIMO Hybrid Precoding Systems
}

Ruiyan Du

Northeastern University - Qinhuangdao Campus

Huajing Liu ( $\sim$ liuhuajing233@163.com )

Northeastern University https://orcid.org/0000-0001-5227-7907

Fulai Liu

Northeastern University - Qinhuangdao Campus

Dan Li

Northeastern University

\section{Research Article}

Keywords: MmWave, Hybrid precoding , Dynamic connected structure , Energy efficiency

Posted Date: June 4th, 2021

DOI: https://doi.org/10.21203/rs.3.rs-513331/v1

License: @ (i) This work is licensed under a Creative Commons Attribution 4.0 International License. Read Full License 


\title{
HP-SFC Algorithm for MmWave Massive MIMO Hybrid Precoding Systems
}

\author{
Ruiyan $\mathbf{D u}^{1,2, *}$ - Huajing $\mathbf{L i u}^{2, *}$. \\ Fulai Liu ${ }^{1,2, *} \cdot \operatorname{Dan} \mathbf{L i}^{2}$
}

Received: date / Accepted: date

\begin{abstract}
This paper presents an alternating iteration hybrid precoding algorithm for the switch network-based dynamic fully-connected (SFC) structure, namely HP-SFC algorithm. Firstly, based on the sparsity of switch network, the optimization problem of the analog switch precoding matrix is transformed into a binary dictionary updating problem, which avoids to deal with the binary constraint straightly. Then, the optimization problem of the analog phase shifter (PS) precoding matrix is modeled as a quadratic unimodular programming problem by the vectorization of the analog PS precoding matrix. So that the analog PS precoding matrix can be readily optimized. Finally, the analog switch precoding matrix, the analog PS precoding matrix and the digital precoding matrix are alternately optimized via the block coordinate descent, the generalized power method and the least square, respectively. Theoretical analysis and simulation results show that the proposed algorithm can provide brilliantly hybrid precoding performence campared with the previous works, for example: 1) it reduces the hybrid precoding matrix residual, so that its spectral efficiency is close to the full digital optimal precoding; 2) it provids at least $15 \%$ energy efficiency improvement comparing with related algorithms.
\end{abstract}

Ruiyan Du

ruiyandu@126.com

Huajing Liu

liuhuajing233@163.com

Fulai Liu

fulailiu@126.com

Fulai Liu

lidan0423@126.com

${ }^{1}$ Engineer Optimization \& Smart Antenna Institute, Northeastern University at Qinhuangdao, Qinhuangdao, China

2 School of Computer Science and Engineering, Northeastern University, Shenyang, China

* Corresponding author: Ruiyan Du, Huajing Liu, Fulai Liu 
Keywords MmWave - Hybrid precoding - Dynamic connected structure · Energy efficiency

\section{Introduction}

Millimeter wave (mmWave) massive multiple-input multiple-output (MIMO) systems relieve the pressure from scarce wireless spectrum resource and attract extensive attentions in recent years [1]. Hybrid precoding is one of the key techniques for mmWave MIMO systems, which offers analogous performance and reduces hardware cost compared with the optimal fully digital precoder [2]. The hybrid precoding structures can be divided into two categories, that is, fully-connected structure and partially-connected structure [3].

In the fully-connected structure, each radio frequency $(\mathrm{RF})$ chain is connected to all antennas, which provides a high spectral efficiency [4]. The fullyconnected precoding is widely studied in recent years. For example, the hybrid precoding problem for fully-connected structure is formulated as a sparse reconstruction problem [5]. A spatially sparse precoding algorithm based on orthogonal matching pursuit (OMP) is proposed as well. Moreover, the thought of alternative optimization is adopted to solve the hybrid precoding problem [6]. A phase extraction-based alternating minimization (PE-AltMin) algorithm is proposed by imposing an orthogonal property of the digital precoder. Nevertheless, the fully-connected structure is burdened with high power consumption due to an enormous number of phase shifters (PSs) are required.

The partially-connected structure possesses lower power consumption compared with the fully-connected structure, since each RF chain is merely connected with a subset of antennas [7]. For partially-connected structure, the hybrid precoder design problem can be considered as a matrix factorization problem and a semidefinite relaxation based AltMin (SDR-AltMin) algorithm is given [8]. Disappointingly, the hybrid precoding performance of the partiallyconnected structure is mediocre.

Benefitted from the low hardware cost and power consumption of switchs, an attractive tradeoff is achieved between the spectral efficiency and the power consumption employing switch network in the analog module of hybrid precoder. Several dynamic connected structures based on switch network are proposed recently. The dynamic partially-connected structure is presented [9], which implements a dynamic connection between RF chains and antenna elements via switch network. Based on the dynamic partially-connected structure, a greedy algorithm is proposed to determine the connection state between RF chains and antenna elements. Further, an exhaustive search-based algorithm is introduced [10], which configures the antenna-to-RF-chain connection state by relocating boundary elements of antenna subsets. The dynamic partiallyconnected structure outperforms the classical partially-connected one since the baseband data stream information is shared by all antenna elements.

However, it is worth to be mentioned that the dynamic partially-connected precoder still takes on a spectral efficiency gap compared with the optimal 
fully digital precoder. In order to further improve the spectral efficiency, a novel dynamic fully-connected structure is studied [11], in which a switch network is added to connect the PSs and antennas. For the switch networkbased dynamic fully-connected (SFC) structure, a two-stage hybrid precoding algorithm is introduced, which separates the hybrid precoding problem into two residual minimization problems and solves in different optimization stage. The two-stage algorithm presents considerable spectral efficiency and energy efficiency, however, the process of sovling two-stage optimization problem may incur large hybrid precoding matrix residuals and low spectral efficiency.

So that, this paper presents an effective hybrid precoding algorithm with the SFC structure, i.e. HP-SFC algorithm. The main contributions are summarized as follows:

- An effective algorithm is proposed based on the SFC structure, which optimizes the analog switch precoding matrix, the analog PS precoding matrix and the digital precoding matrix alternatively and iteratively. Based on the compressed sensing theory, the analog switch precoding matrix optimization problem can be refomulated as a dictionary update problem and solved by block coodinate descent effectively. The objective function of the analog PS precoding matrix optimazition problem is reformulated as a quadratic form by the matrix vectorize property, so that a KarushKuhn-Tucker (KKT) solution can be effortlessly obtained. Then, the digital precoding matrix is given by the least square method.

- Several comparisons are provided between the proposed algorithm and related algorithms with different structures. Simulation results show that the proposed algorithm presents attractive spectral efficiency. Concurrently, since switch network enioys low hardware cost and power consumption, the proposed algorithm contributes decent energy efficiency.

The remainder of the paper is organized as follows. The hybrid precoding signal model and the SFC structure are introduced in Section 2. Section 3 introduces the proposed hybrid precoding algorithm detailedly. Section 4 gives several simulation results to demonstrate the performance of the proposed algorithm. Finally, the conclusion is presented in Section 5.

Notations : In the following, the bold upper-case letters and bold lowercase letters denote matrix and vector respectively. The field of complex number is represented by $\mathbb{C} . \boldsymbol{A}_{i j}$ is the $(i, j)$ th element of $\boldsymbol{A} \cdot \boldsymbol{A}^{\mathrm{T}}, \boldsymbol{A}^{*}, \boldsymbol{A}^{\mathrm{H}}$ and $\boldsymbol{A}^{-1}$ represent the transpose, conjugate, conjugate transpose and inverse of $\boldsymbol{A} .|\boldsymbol{A}|$, $\|\boldsymbol{A}\|_{F}$ and $\|\boldsymbol{A}\|_{2}$ denote the determinant, Frobenius norm and $l_{2}$ norm of $\boldsymbol{A}$. $\angle\{\boldsymbol{A}\}$ is taken to mean a matrix containing the phases of the entries of $\boldsymbol{A}$. $\boldsymbol{I}_{N}$ indicates the $N \times N$ identity matrix. $\otimes$ denotes Kronecker product, $\operatorname{tr}\{\cdot\}$ and $\operatorname{vec}\{\cdot\}$ stand for trace and vectorization operator. $\mathcal{C N}(a, b)$ is the complex Gaussian distribution with the mean $a$ and the covariance $b$. 


\section{System model}

\subsection{Signal model}

Consider a single-user mmWave massive MIMO system, in which $N_{s}$ independent baseband data streams are send from a transmitter with $N_{t}$ antennas and $N_{R F}$ RF chains to a receiver with $N_{r}$ antennas and $N_{R F}$ RF chains. Without loss of generality, a narrow-band block-fading channel model is adopted to describe the transmission environment between the transmitter and receiver. Thus, the received baseband signal $\boldsymbol{y}$ is expressed as

$$
\boldsymbol{y}=\sqrt{\rho} \boldsymbol{W}^{\mathrm{H}} \boldsymbol{H} \boldsymbol{F} \boldsymbol{s}+\boldsymbol{W}^{\mathrm{H}} \boldsymbol{n}
$$

where $\rho$ is the average received power, $\boldsymbol{H} \in \mathbb{C}^{N_{r} \times N_{t}}$ denotes the channel matrix, $\boldsymbol{W} \in \mathbb{C}^{N_{s} \times N_{r}}$ and $\boldsymbol{F} \in \mathbb{C}^{N_{t} \times N_{s}}$ represents the hybrid combining matrix and the hybrid precoding matrix, respectively. Furthermore, $s=\left[s_{1}, \cdots, s_{N_{s}}\right]^{\mathrm{T}}$ stands for the transmit signal vector such that $\mathbf{E}\left[\boldsymbol{s} \boldsymbol{s}^{\mathrm{H}}\right]=\frac{1}{N_{s}} \boldsymbol{I}_{N_{s}}$, and $\boldsymbol{n}$ is the independent and identically distributed (i.i.d.) complex Gaussian noise vector with $\mathcal{C} \mathcal{N}\left(0, \sigma_{n}^{2}\right)$, in which $\sigma_{n}^{2}$ stands for the noise power.

Based on the Saleh-Valenzuela channel model [12], the channel matrix $\boldsymbol{H}$ consists a sum of $N_{c l}$ scattering clusters, each of which composes of $N_{\text {ray }}$ rays. So that it can be written as

$$
\boldsymbol{H}=\sqrt{\frac{N_{t} N_{r}}{L}} \sum_{i=1}^{N_{c l}} \sum_{k=1}^{N_{r a y}} \alpha_{i k} \boldsymbol{a}_{\mathrm{r}}\left(\phi_{i k}^{r}, \theta_{i k}^{r}\right) \boldsymbol{a}_{\mathrm{t}}^{\mathrm{H}}\left(\phi_{i k}^{t}, \theta_{i k}^{t}\right)
$$

where the total number of propagation paths $L=N_{c l} N_{r a y}, \alpha_{i k}$ is the complex gain of the $k$ th ray in the $i$ th scattering cluster. $\phi_{i k}^{t}\left(\theta_{i k}^{t}\right)$ and $\phi_{i k}^{r}\left(\theta_{i k}^{r}\right)$ are the azimuth (elevation) angle of departure (AOD) and the azimuth (elevation) angle of arrival (AOA) associated with the $k$ th ray in $i$ th cluster, respectively. $\boldsymbol{a}_{t}\left(\phi_{i k}^{t}, \theta_{i k}^{t}\right)$ and $\boldsymbol{a}_{r}\left(\phi_{i k}^{r}, \theta_{i k}^{r}\right)$ stand for the array response vector of the transmitter and receiver.

For an $M \times N$ uniform planar array (UPA) with the half-wavelength interelement space, the array response vector is given as

$\boldsymbol{a}_{\mathrm{UPA}}(\phi, \theta)=\frac{1}{\sqrt{M N}}\left[\begin{array}{llll}1 & \cdots & e^{j \pi(m \sin \phi \sin \theta+n \cos \theta)} \cdots & e^{j \pi((M-1) \sin \phi \sin \theta+(N-1) \cos \theta)}\end{array}\right]^{\mathrm{T}}$

where $0 \leq m \leq M-1$ and $0 \leq n \leq N-1$.

With the assumption that the perfect channel state information (CSI) is known at both the transmitter and receiver, when Gaussian symbols are transmitted over the mmWave channel, the spectral efficiency achieved is given by

$$
R=\log _{2}\left(\left|\boldsymbol{I}_{N_{s}}+\frac{\rho}{N_{s}} \boldsymbol{R}_{n}^{-1} \boldsymbol{W}^{\mathrm{H}} \boldsymbol{H} \boldsymbol{F} \boldsymbol{F}^{\mathrm{H}} \boldsymbol{H}^{\mathrm{H}} \boldsymbol{W}\right|\right)
$$

where the noise covariance matrix after combining $\boldsymbol{R}_{n}=\sigma_{n}^{2} \boldsymbol{W}^{\mathrm{H}} \boldsymbol{W}$. 


\subsection{SFC structure}

In this subsection, the SFC structure and the hybrid precoding problem based on SFC structure are described detailedly.

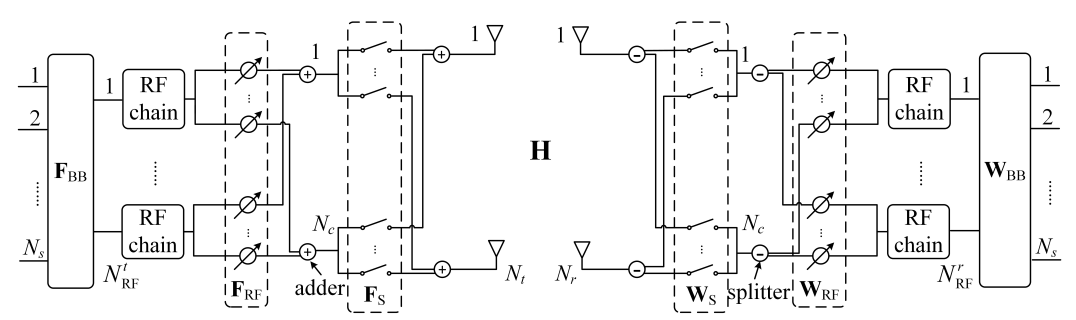

Fig. 1: Single-user mmWave MIMO system based on the SFC structure.

The SFC structure for the single-user mmWave MIMO system is shown in Fig.1, in which the analog precoding (combining) is implemented both PSs and switch network. It can be seen that the signal from each RF chain is processed by $N_{c}$ PSs, where $N_{c} \ll N_{t}$, so that $N_{R F} N_{c}$ PSs are equipped in the SFC structure.

Hence, the precoding matrix $\boldsymbol{F}$ and the combining matrix $\boldsymbol{W}$ of the SFC structure are expressed as follows

$$
\begin{aligned}
\boldsymbol{F} & =\boldsymbol{F}_{S} \boldsymbol{F}_{P S} \boldsymbol{F}_{B B} \\
\boldsymbol{W} & =\boldsymbol{W}_{S} \boldsymbol{W}_{P S} \boldsymbol{W}_{B B} .
\end{aligned}
$$

where $\boldsymbol{F}_{B B} \in \mathbb{C}^{N_{R F} \times N_{s}}, \boldsymbol{F}_{P S} \in \mathbb{C}^{N_{c} \times N_{R F}}$ and $\boldsymbol{F}_{S} \in \mathbb{C}^{N_{t} \times N_{c}}$ denotes the digital precoding matrix, the analog PS precoding matrix and the analog switch precoding matrix, $\boldsymbol{W}_{B B} \in \mathbb{C}^{N_{R F} \times N_{s}}, \boldsymbol{W}_{P S} \in \mathbb{C}^{N_{c} \times N_{R F}}$ and $\boldsymbol{W}_{S} \in \mathbb{C}^{N_{r} \times N_{c}}$ denote the digital combining matrix, the analog PS combining matrix and the analog switch combining matrix, respectively.

Consider the hardware characteristic of switches, the analog switch precoding matrix $\boldsymbol{F}_{S}$ and the analog switch combining matrix $\boldsymbol{W}_{S}$ are subject to the binary constraint, that is $\boldsymbol{F}_{S, i j} \in\{0,1\}$ and $\boldsymbol{W}_{S, i j} \in\{0,1\}$. Moreover, the analog PS precoding matrix $\boldsymbol{F}_{P S}$ and the analog PS combining matrix $\boldsymbol{W}_{P S}$ are subject to the unit modulus constraint, namely, $\left|\boldsymbol{F}_{P S, m n}\right|=1$ and $\left|\boldsymbol{W}_{P S, m n}\right|=1$.

On the basis of [5], the hybrid precoding (combining) problem based on the SFC structure can be approximately divided into two subproblems as follows

$$
\begin{aligned}
\min _{\boldsymbol{F}_{S}, \boldsymbol{F}_{P S}, \boldsymbol{F}_{B B}} & \left\|\boldsymbol{F}_{\mathrm{opt}}-\boldsymbol{F}_{S} \boldsymbol{F}_{P S} \boldsymbol{F}_{B B}\right\|_{F}^{2} \\
\text { s.t. } & \boldsymbol{F}_{S, i j} \in\{0,1\}, \quad i \in\left[1, N_{t}\right], j \in\left[1, N_{c}\right] \\
& \left|\boldsymbol{F}_{P S, m n}\right|=1, \quad m \in\left[1, N_{c}\right], n \in\left[1, N_{R F}\right] \\
& \left\|\boldsymbol{F}_{S} \boldsymbol{F}_{P S} \boldsymbol{F}_{B B}\right\|_{F}^{2}=N_{s}
\end{aligned}
$$




$$
\begin{aligned}
\min _{\boldsymbol{W}_{S}, \boldsymbol{W}_{P S}, \boldsymbol{W}_{B B}} & \left\|\boldsymbol{W}_{\mathrm{opt}}-\boldsymbol{W}_{S} \boldsymbol{W}_{P S} \boldsymbol{W}_{B B}\right\|_{F}^{2} \\
\text { s.t. } & \boldsymbol{W}_{S, i j} \in\{0,1\}, \quad i \in\left[1, N_{r}\right], j \in\left[1, N_{c}\right] \\
& \left|\boldsymbol{W}_{P S, m n}\right|=1, \quad m \in\left[1, N_{c}\right], n \in\left[1, N_{R F}\right]
\end{aligned}
$$

where $\left\|\boldsymbol{F}_{S} \boldsymbol{F}_{P S} \boldsymbol{F}_{B B}\right\|_{F}^{2}=N_{s}$ denotes the normalized transmit power constraint.

According to [13], the optimal fully digital precoding matrix and combining matrix are formulated as $\boldsymbol{F}_{\mathrm{opt}}=\boldsymbol{V}_{:, 1: N_{s}}$ and $\boldsymbol{W}_{\mathrm{opt}}=\boldsymbol{U}_{:, 1: N_{s}}$ respectively, where the SVD of the mmWave channel matrix $\boldsymbol{H}=\boldsymbol{U} \boldsymbol{\Sigma} \boldsymbol{V}^{\mathrm{H}}$.

Noticeably, the precoding problem (6) and the combining problem (7) have similar mathematical expressions, so that the remaining of this paper will mainly focus on the precoding problem (6), and the combining problem (7) can be similarly tackled using the proposed method.

\section{Hybrid precoding strategy}

The joint optimization problem (6) is difficult to be solved straightly, due to the unit modulus constraint of the analog PS precoding matrix $\boldsymbol{F}_{P S}$ and the binary constraint of the analog switch precoding matrix $\boldsymbol{F}_{S}$. In this section, the HP-SFC algorithm is proposed to optimize the analog switch precoding matrix $\boldsymbol{F}_{S}$, the analog PS precoding matrix $\boldsymbol{F}_{P S}$ and the digital precoding matrix $\boldsymbol{F}_{B B}$ respectively.

\subsection{Optimization of analog switch precoding matrix}

In this subsection, suppose that the analog PS precoding matrix $\boldsymbol{F}_{P S}$ and the digital precoding matrix $\boldsymbol{F}_{B B}$ are known, the optimization problem of the analog switch precoding matrix $\boldsymbol{F}_{S}$ can be rewritten as

$$
\begin{aligned}
& \min _{\boldsymbol{F}_{S}}\left\|\boldsymbol{F}_{\mathrm{opt}}-\boldsymbol{F}_{S} \boldsymbol{F}_{\mathrm{eff}}\right\|_{F}^{2} \\
& \text { s.t. } \boldsymbol{F}_{S, i j} \in\{0,1\}, \quad i \in\left[1, N_{t}\right], j \in\left[1, N_{c}\right]
\end{aligned}
$$

where the effective precoding matrix $\boldsymbol{F}_{\text {eff }}$ is defined as the product of the analog PS precoding matrix $\boldsymbol{F}_{P S}$ and the digital precoding matrix $\boldsymbol{F}_{B B}$.

Based on the compressed sensing theory [14], the analog switch precoding matrix $\boldsymbol{F}_{S}$ can be considered as a sparse representation dictionary which satisfies the binary constraint. Moreover, the optimization problem (8) can be viewed as a dictionary update problem and solved by the block coodinate descent method.

Expand the product of the analog switch precoding matrix $\boldsymbol{F}_{S}$ and the effective precoding matrix $\boldsymbol{F}_{\text {eff }}$, the objective function of the optimization problem (8) is represented as $\left\|\boldsymbol{F}_{\text {opt }}-\sum_{j=1}^{N_{c}} \boldsymbol{f}_{S, j} \boldsymbol{f}_{\mathrm{eff}, j}^{\mathrm{H}}\right\|_{F}^{2}$, where $\boldsymbol{f}_{S, j} \in \mathbb{C}^{N_{t} \times 1}$ and $f_{\text {eff }, j}^{\mathrm{H}} \in \mathbb{C}^{1 \times N_{s}}$ stand for the $j$ th column of the analog switch precoding matrix $\boldsymbol{F}_{S}$ and the $j$ th row of the effective precoding matrix $\boldsymbol{F}_{\text {eff }}$ respectively. 
Assume that $\boldsymbol{f}_{S, k}(k \neq j)$ and $\boldsymbol{f}_{\text {eff, }, k}^{\mathrm{H}}(k \neq j)$ are fixed, merely focus on the $j$ th column of $\boldsymbol{F}_{S}$ and the $j$ th row of $\boldsymbol{F}_{\text {eff }}$, problem (8) can be rewritten as

$$
\begin{aligned}
& \min _{\boldsymbol{F}_{S}}\left\|\boldsymbol{Y}-\boldsymbol{f}_{S, j} \boldsymbol{f}_{\mathrm{eff}, j}^{\mathrm{H}}\right\|_{F}^{2} \\
& \text { s.t. } \boldsymbol{F}_{S, i j} \in\{0,1\}, \quad i \in\left[1, N_{t}\right], j \in\left[1, N_{c}\right]
\end{aligned}
$$

where $\boldsymbol{Y}=\boldsymbol{F}_{\mathrm{opt}}-\sum_{k \neq j} \boldsymbol{f}_{S, k} \boldsymbol{f}_{\mathrm{eff}, k}$.

It is deduced that the problem (9) can be divided into the following $N_{t}$ independent subproblems

$$
\begin{aligned}
& \min _{\boldsymbol{F}_{S, i j}}\left\|\boldsymbol{y}_{i}^{\mathrm{H}}-\boldsymbol{F}_{S, i j} \boldsymbol{f}_{\mathrm{eff}, j}^{\mathrm{H}}\right\|_{2}^{2} \\
& \text { s.t. } \boldsymbol{F}_{S, i j} \in\{0,1\}, \quad i \in\left[1, N_{t}\right]
\end{aligned}
$$

where $\boldsymbol{y}_{i}^{\mathrm{H}}$ is the $i$ th row of $\boldsymbol{Y}$.

Hence, the entries of the analog switch precoding matrix $\boldsymbol{F}_{S}$ can be singly optimized as

$$
\boldsymbol{F}_{S, i j}=\left\{\begin{array}{ll}
0, & \left\|\boldsymbol{y}_{i}^{\mathrm{H}}\right\|_{2}^{2} \leq\left\|\boldsymbol{y}_{i}^{\mathrm{H}}-\boldsymbol{f}_{\text {eff, }}^{\mathrm{H}}\right\|_{2}^{2} \\
1, & \left\|\boldsymbol{y}_{i}^{\mathrm{H}}\right\|_{2}^{2}>\left\|\boldsymbol{y}_{i}^{\mathrm{H}}-\boldsymbol{f}_{\mathrm{eff}, j}^{\mathrm{H}}\right\|_{2}^{2}
\end{array} .\right.
$$

Exploiting (11), the entries of the analog switch precoding matrix $\boldsymbol{F}_{S}$ can be separately optimized.

3.2 Optimization of analog PS precoding matrix

The analog PS precoding matrix $\boldsymbol{F}_{P S}$ is optimized in this subsection, the optimization problem of which is given by

$$
\begin{aligned}
& \min _{\boldsymbol{F}_{P S}}\left\|\boldsymbol{F}_{\mathrm{opt}}-\boldsymbol{F}_{S} \boldsymbol{F}_{P S} \boldsymbol{F}_{B B}\right\|_{F}^{2} \\
& \text { s.t. }\left|\boldsymbol{F}_{P S, m n}\right|=1, \quad m \in\left[1, N_{c}\right], n \in\left[1, N_{R F}\right]
\end{aligned}
$$

where the analog switch precoding matrix $\boldsymbol{F}_{S}$ and the digital precoding matrix $\boldsymbol{F}_{B B}$ are assumed to be fixed.

Based on the matrix vectorize property $\operatorname{vec}(\boldsymbol{A} \boldsymbol{X} \boldsymbol{B})=\left(\boldsymbol{B}^{\mathrm{T}} \otimes \boldsymbol{A}\right) \operatorname{vec}(\boldsymbol{X})$, the objective function of the optimization problem (12) can be transformed into $\left\|\boldsymbol{f}_{\mathrm{opt}}-\left(\boldsymbol{F}_{B B}^{\mathrm{T}} \otimes \boldsymbol{F}_{S}\right) \boldsymbol{f}_{P S}\right\|_{F}^{2}$, where $\boldsymbol{f}_{\mathrm{opt}}=\operatorname{vec}\left(\boldsymbol{F}_{\mathrm{opt}}\right), \boldsymbol{f}_{P S}=\operatorname{vec}\left(\boldsymbol{F}_{P S}\right)$.

Moreover, the Frobenius norm minimization problem (12) can be rewritten as

$$
\begin{aligned}
& \max _{\boldsymbol{f}_{P S}} \tilde{\boldsymbol{f}}_{P S}^{\mathrm{H}}(-\boldsymbol{B}) \tilde{\boldsymbol{f}}_{P S} \\
& \text { s.t. }\left|\boldsymbol{f}_{P S}(o)\right|=1, \quad o \in\left[1, N_{c} N_{R F}\right]
\end{aligned}
$$

where

$$
\boldsymbol{B}=\left[\begin{array}{cc}
\left(\boldsymbol{F}_{B B}^{*} \otimes \boldsymbol{F}_{S}^{\mathrm{H}}\right)\left(\boldsymbol{F}_{B B}^{\mathrm{T}} \otimes \boldsymbol{F}_{S}\right) & -\left(\boldsymbol{F}_{B B}^{\mathrm{T}} \otimes \boldsymbol{F}_{S}\right)^{\mathrm{H}} \boldsymbol{f}_{\mathrm{opt}} \\
-\boldsymbol{f}_{\mathrm{opt}}^{\mathrm{H}}\left(\boldsymbol{F}_{B B}^{\mathrm{T}} \otimes \boldsymbol{F}_{S}\right) & 0
\end{array}\right] \text { and } \tilde{\boldsymbol{f}}_{P S}=\left[\begin{array}{ll}
\boldsymbol{f}_{P S} & 1
\end{array}\right]^{\mathrm{T}} .
$$


A KKT solution of the quadratic unimodular programming problem (13) can be obtained by the iteratively generalized power method [15], namely

$$
\boldsymbol{F}_{P S}^{(l)}=e^{j \angle\left(\boldsymbol{F}_{P S}^{(l-1)}-\frac{1}{p}\left(\boldsymbol{M}_{S} \boldsymbol{F}_{P S}^{(l-1)} \boldsymbol{M}_{B B}-\boldsymbol{M}_{o}\right)\right)} e^{-j \angle\left(1+\frac{1}{p} \operatorname{tr}\left\{\boldsymbol{M}_{o}^{\mathrm{H}} \boldsymbol{F}_{P S}^{(l-1)}\right\}\right)}
$$

where $l$ denotes the iteration index, $\boldsymbol{M}_{B B}=\boldsymbol{F}_{B B} \boldsymbol{F}_{B B}^{\mathrm{H}}, \boldsymbol{M}_{S}=\boldsymbol{F}_{S}^{\mathrm{H}} \boldsymbol{F}_{S}, \boldsymbol{M}_{o}=$ $\boldsymbol{F}_{S}^{\mathrm{H}} \boldsymbol{F}_{\mathrm{opt}} \boldsymbol{F}_{B B}^{\mathrm{H}}$ and $p=\frac{\operatorname{tr}\left\{\boldsymbol{M}_{B B}\right\} \operatorname{tr}\left\{\boldsymbol{M}_{S}\right\}}{N_{c} N_{R F}+1}+\left(\frac{N_{c} N_{R F}}{N_{c} N_{R F}+1}\left(\left\|\boldsymbol{M}_{B B}\right\|_{F}^{2}\left\|\boldsymbol{M}_{S}\right\|_{F}^{2}+2\left\|\boldsymbol{M}_{o}\right\|_{F}^{2}-\right.\right.$ $\left.\left.\frac{\operatorname{tr}\left\{M_{B B}\right\}^{2} \operatorname{tr}\left\{M_{S}\right\}^{2}}{N_{c} N_{R F}+1}\right)\right)^{\frac{1}{2}}$.

\subsection{Optimization of digital precoding matrix}

Based on the analog switch precoding matrix $\boldsymbol{F}_{S}$ and the analog PS precoding matrix $\boldsymbol{F}_{P S}$ optimized above, a near optimal analytical solution of the digital precoding matrix $\boldsymbol{F}_{B B}$ can be given by the least square, that is

$$
\boldsymbol{F}_{B B}=\left(\boldsymbol{F}_{P S}^{\mathrm{H}} \boldsymbol{F}_{S}^{\mathrm{H}} \boldsymbol{F}_{S} \boldsymbol{F}_{P S}\right)^{-1} \boldsymbol{F}_{P S}^{\mathrm{H}} \boldsymbol{F}_{S}^{\mathrm{H}} \boldsymbol{F}_{\mathrm{opt}} \cdot
$$

where the analog switch precoding matrix $\boldsymbol{F}_{S}$ and the analog PS precoding matrix $\boldsymbol{F}_{P S}$ are presumed to be known.

Additionally, in order to satisfy the transmit power constraint $\left\|\boldsymbol{F}_{S} \boldsymbol{F}_{P S} \boldsymbol{F}_{B B}\right\|_{F}^{2}=$ $N_{s}$, the digital precoding matrix $\boldsymbol{F}_{B B}$ have to be multiplied by a coefficient $\frac{\sqrt{N_{S}}}{\left\|\boldsymbol{F}_{S} \boldsymbol{F}_{P S} \boldsymbol{F}_{B B}\right\|_{F}}$.

Based on the above discussion, the proposed algorithm is summarized in Table 1.

Table 1: HP-SFC algorithm

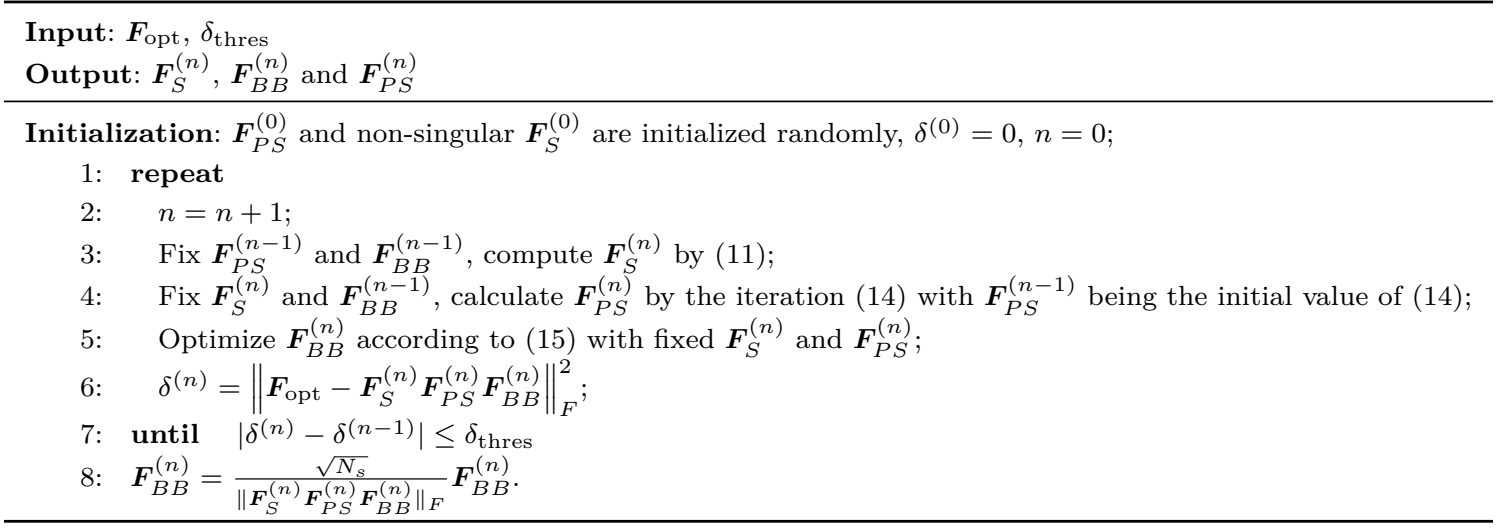




\section{Simulation results}

In this section, several simulation results are provided to evaluate the performance of the proposed HP-SFC algorithm. For comparison, the spectral efficiencies and energy efficiencies of the HP-SFC algorithm and related algorithms are analyzed detailedly, such as the two-stage algorithm with the SFC structure [11], the OMP-based sparse hybrid precoding algorithm with fully-connected structure [5], and the SDR-AltMin algorithm with partialiyconnected structure [8].

Energy efficiency is one of the performance indexs for mmWave hybrid precoding systems, which integrates spectral efficiency and power consumption into account concurrently. The energy efficiency $\eta$ is defined as

$$
\eta=\frac{R}{P}
$$

where $R$ indicates the spectral efficiency, $P$ indicates the total power consumption, i.e.

$$
P= \begin{cases}P_{t}+P_{R F} N_{R F}+P_{P S} N_{R F} N_{c}+P_{S} N_{c} N_{t}, & \text { Two }- \text { stage and HP }- \text { SFC } \\ P_{t}+P_{R F} N_{R F}+P_{P S} N_{R F} N_{t}, & \text { OMP }- \text { based } \\ P_{t}+P_{R F} N_{R F}+P_{P S} N_{t}, & \text { SDR }- \text { AltMin }\end{cases}
$$

where $P_{t}$ signifies the transmit power, $P_{R F}, P_{P S}$ and $P_{S}$ denote the power consumption of RF chain, PS and switch, respectively. Additionally, the power consumption in the following simulation is set as $P_{t}=1 \mathrm{~W}, P_{R F}=250 \mathrm{~mW}$, $P_{P S}=50 \mathrm{~mW}$, and $P_{S}=5 \mathrm{~mW}[16][17]$.

In order to investigate the hybrid precoding performance of all schemes, it is suppose that the transmitter and receiver are equipped with UPAs. The channel matrix $\boldsymbol{H}$ satisfies the Saleh-Valenzuela channel model above mentioned in Section 2. The mmWave channel consists of $N_{c l}=5$ clusters and each cluster comprises $N_{\text {ray }}=10$ rays, i.e., the total number of paths $L=N_{c l} N_{\text {ray }}=50$. The mean azimuth AOD (AOA) and elevation AOD (AOA) of each cluster are uniformly distributed in $[0,2 \pi)$. In clusters, the azimuth AOD (AOA) and elevation AOD (AOA) follow the Laplace distribution with a 10-degree angular spread. Meanwhile, the complex gain of each path is assumed to follow the standard complex normal distribution $\mathcal{C N}(0,1)$. All simulation results are obtained by taking the average of 1000 random channel realizations.

Firstly, the impact of $N_{c}$ on the prcoding performance of the proposed HP-SFC algorithm is shown in Fig.2 and Fig.3, where $N_{s}=N_{R F}=4, N_{t}=$ $144, N_{r}=36, \mathrm{SNR}=10 \mathrm{~dB}$. It is clear that the spectral efficiencies of the proposed algorithm and the two-stage algorithm in Fig. 2 grow dramatically as $N_{c}$ increases and approximatively reaches a stable status when $N_{c} \geq 36$. The proposed algorithm surpasses the OMP-based algorithm and the twostage algorithm when $N_{c} \geq 12$. Moreover, the proposed algorithm provides a 


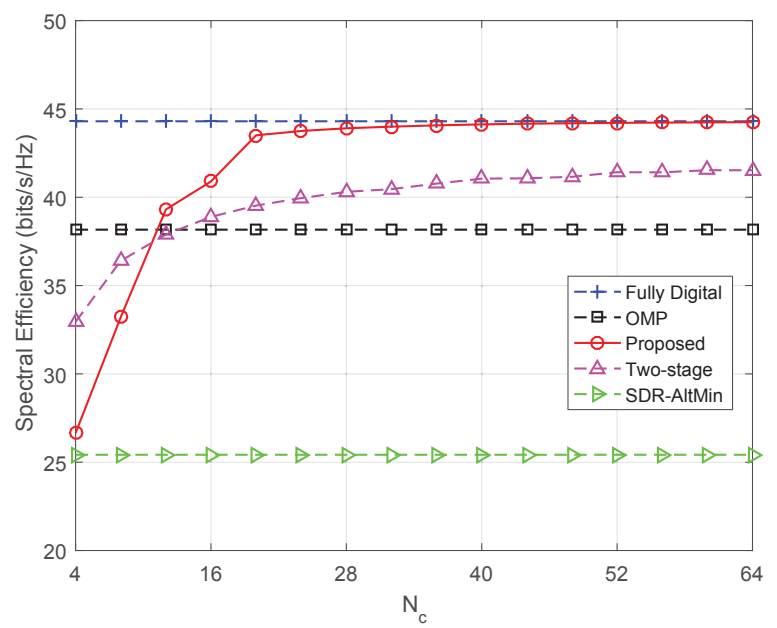

Fig. 2: Spectral efficiencies versus $N_{c}$.

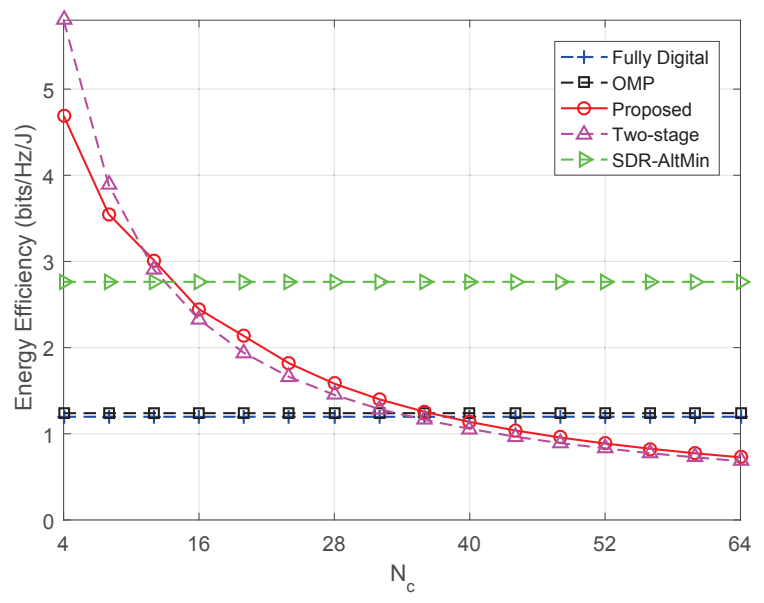

Fig. 3: Energy efficiencies versus $N_{c}$.

comparable energy efficiency with the optimal fully digital scheme when $N_{c}$ is large enough, which verifies the validity of the proposed algorithm.

Fig.3 provides the energy efficiencies of the proposed algorithm and contrast schemes. The energy efficiencies of the proposed algorithm and the twostage algorithm decrease with $N_{c}$ due to more PSs and switches are required with a large number of $N_{c}$, which lead to high power consumption. It is observed that the proposed algorithm outperforms the two-stage algorithm when $N_{c} \geq 12$. Moreover, compare to the OMP-based algorithm, the proposed algo- 
rithm prsents higher energy efficiency when $N_{c} \leq 36$. The SDR-AltMin algorithm presents a preferable energy efficiency, however, the partialy-connected structure results in the limited spectral efficiency as shown in Fig.2.

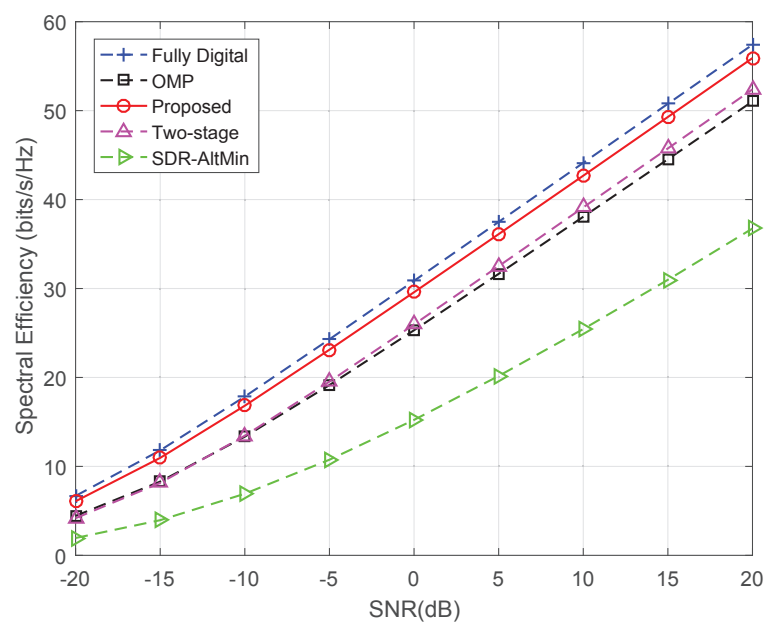

Fig. 4: Spectral efficiencies versus SNR.

In Fig.4, the spectral efficiencies of all schemes as the functions of signalto-noise ratio (SNR) are detailly exhibited, where $N_{s}=N_{R F}=4, N_{t}=144$, $N_{r}=36, N_{c}=20$. It can be seen that the spectral efficiency of the proposed algorithm approachs the optimal fully digital scheme with only $1 \mathrm{bits} / \mathrm{s} / \mathrm{Hz}$ performance gap. When $N_{c}=20$, the power dissipated by the SFC structure equals to $18.4 \mathrm{~W}$, however, that of the fully-connected structure comes up to $30.8 \mathrm{~W}$. Hence, the proposed algorithm with the SFC structure is able to obtain prominent hybrid precoding perfoemance while maintaining lower power consumption.

Fig.5 analyzes the spectral efficiency versus $N_{R F}$ for all schemes with the parameters set as $N_{s}=N_{R F}, N_{t}=144, N_{r}=36, \mathrm{SNR}=10 \mathrm{~dB}$. It is shown that the proposed algorithm offers about $3 \mathrm{bits} / \mathrm{s} / \mathrm{Hz}$ gain compared with the twostage algorithm when $N_{R F}=4$. Meanwhile, the proposed algorithm provides comparable performance with the optimal fully digital scheme when $N_{R F} \leq 3$, which shows the superiority of the proposed algorithm under less RF chain condition.

Fig.6 shows the impact of $N_{R F}$ on the energy efficiency of the proposed algorithm, where $N_{s}=N_{R F}, N_{t}=144, N_{r}=36, \mathrm{SNR}=10 \mathrm{~dB}$. It is evident that as $N_{R F}$ increases, the energy efficiencies of the proposed algorithm, the two-stage algorithm and the SDR-AltMin algorithm grow steadily, however, that of the OMP-based algorithm declines gradually. In spite of the proposed algorithm ranks second to the SDR-AltMin algorithm, the proposed algorithm 


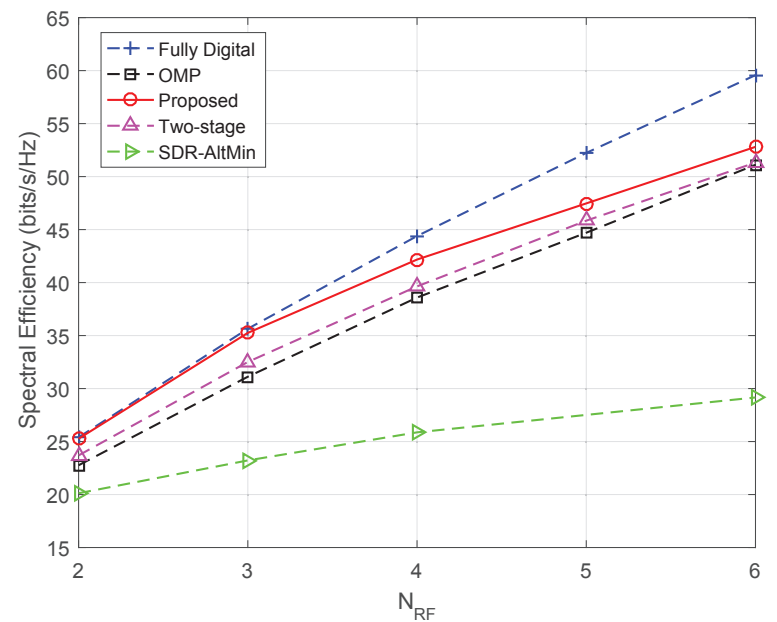

Fig. 5: Spectral efficiencies versus $N_{R F}$.

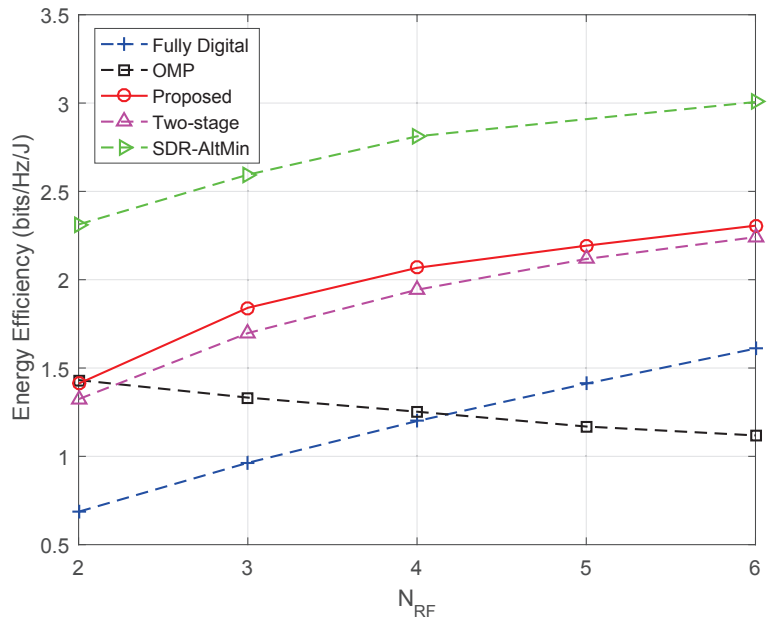

Fig. 6: Energy efficiencies versus $N_{R F}$.

represents prominent hybrid precoding performance since a near optimal spectral efficiency is obtained.

Fig.7 and Fig.8 show the spectral efficiency and the energy efficiency comparisons of the proposed algorithm with other schemes as function of the antennas at transimitter $N_{t}$, where $N_{s}=N_{R F}=4, N_{r}=36, \mathrm{SNR}=10 \mathrm{~dB}$. It can be seen from Fig.7 that the spectral efficiency of all schemes increase with $N_{t}$. Meanwhile, the proposed algorithm provides about $4 \mathrm{bits} / \mathrm{s} / \mathrm{Hz}$ and 


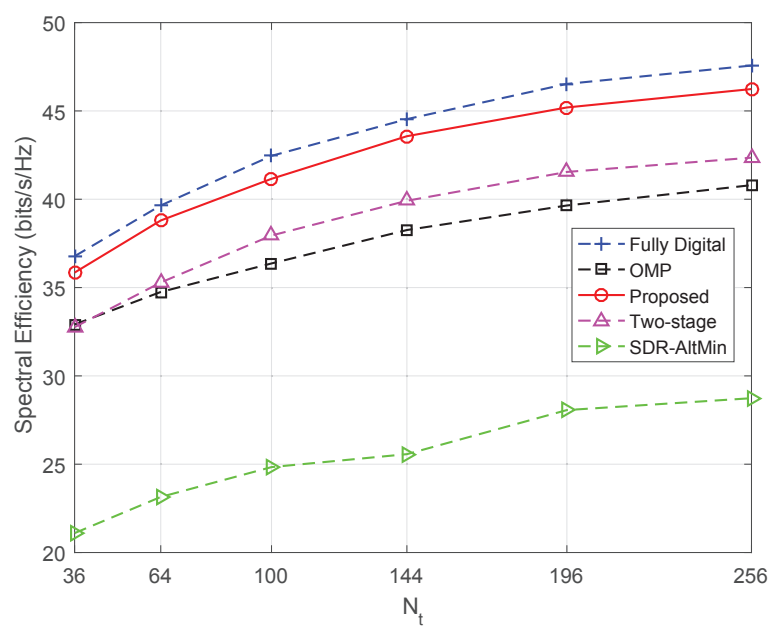

Fig. 7: Spectral efficiencies versus $N_{t}$.

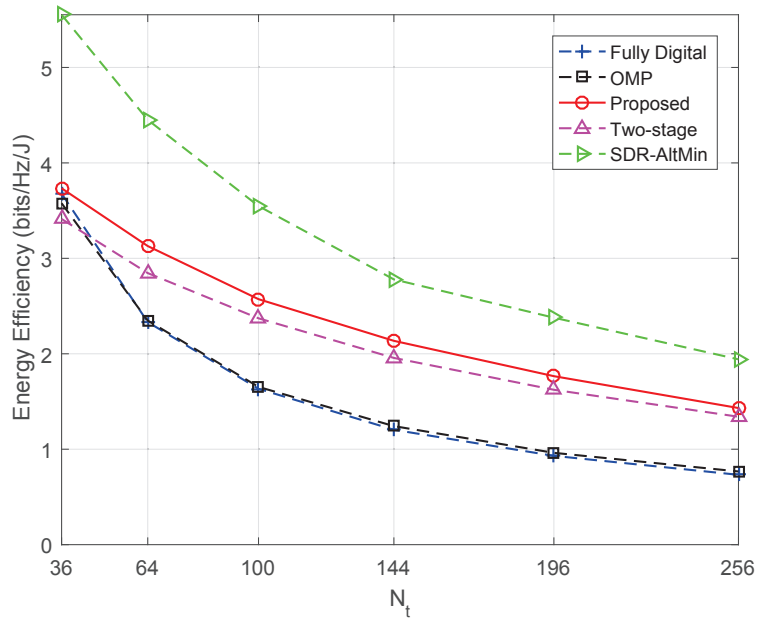

Fig. 8: Energy efficiencies versus $N_{t}$.

$5 \mathrm{bits} / \mathrm{s} / \mathrm{Hz}$ spectral efficiency gap campared with the two-stage algorithm and the OMP-based algorithm when $N_{t} \geq 144$, which demonstrates that the proposed algorithm is a competitive hybrid precoding scheme.

With the increase of $N_{t}$, it is evident that the energy efficiencies decrease rapidly in Fig. 8 since large $N_{t}$ places power consumption burden on the hybrid precoding systems. Moreover, the energy efficiency of the HP-SFC algorithm outperforms the two-stage algorithm with SFC structure, the OMP-based al- 
gorithm with fully-connected structure and the optimal fully digital scheme in all the cases. Compare with the two-stage algorithm, the proposed algorithm improves energy efficiency by about $15 \%$ when $N_{t}=144$. Based on the above discussion, it is demonstrated that the proposed algorithm with SFC structure provides a reasonable tradeoff between spectral efficiency and power consumption.

\section{Conclusion}

This paper focuses on the hybrid precoder design for SFC structure. An effective hybrid precoding algorithm is introduced, which optimizes the precoding matrixes alternatively and iteratively. The analog switch precoding matrix, the analog PS precoding matrix and the digital precoding matrix are derived from the block coodinate descent, iteratively generalized power method and least squares, respectively. Simulation results show that the proposed algorithm provides considerable spectral efficiency. Meanwhile, a considerable energy efficiency improvement is supplied by the proposed algorithm.

Acknowledgements This work was supported by the National Natural Science Foundation of China (Grant No. 61971117) and by the Natural Science Foundation of Hebei Province (Grant No. F2020501007).

\section{Conflict of interest}

The authors declare that they have no conflict of interest regarding the publication of this paper.

\section{References}

1. Zhang, L., Gui, L., Ying, K., \& Qin, Q. (2019). Clustering based hybrid precoding design for multi-user massive MIMO systems. IEEE Transactions on Vehicular Technology, 68(12), 12164-12178.

2. Ahmed, I., Khammari, H., Shahid, A., Musa, A., Kim, K. S., Poorter, E. D., \& Moerman, I. (2018). A survey on hybrid beamforming techniques in 5G: architecture and system model perspectives. IEEE Communications Surveys \& Tutorials, 20(4), 3060-3097.

3. Han, S., I, C., Xu, Z., \& Rowell, C. (2015). Large-scale antenna systems with hybrid analog and digital beamforming for millimeter wave 5G. IEEE Communications Magazine, $53(1), 186-194$

4. Li, M., Wang, Z., Li, H., Liu, Q., \& Zhou, L. (2019). A hardware-efficient hybrid beamforming solution for mmWave MIMO systems. IEEE Wireless Communications, 26(1), $137-143$.

5. Ayach, O. E., Rajagopal, S., Abu-Surra, S., Pi, Z., \& Heath, R. W. (2014). Spatially sparse precoding in millimeter wave MIMO systems. IEEE Transactions on Wireless Communications, 13(3), 1499-1513.

6. Yu, X., Shen, J., Zhang, J., \& Letaief, K. B. (2016). Alternating minimization algorithms for hybrid precoding in millimeter wave MIMO systems. IEEE Journal of Selected Topics in Signal Processing, 10, no. 3, 485-500. 
7. Zhang, Z., Wu, X., \& Liu, D. (2020). Joint precoding and combining design for hybrid beamforming systems with subconnected structure. IEEE Systems Journal, 14(1), 184195

8. Yu, X., Shen, J., Zhang, J., \& Letaief,K. B. (2016). Alternating minimization algorithms for hybrid precoding in millimeter wave MIMOsystems. IEEE Journal of Selected Topics in Signal Processing, 10(3), 485-500.

9. Park, S., Alkhateeb, A., \& Heath, R. W. (2017). Dynamic subarrays for hybrid precoding in wideband mmWave MIMO systems. IEEE Transactions on Wireless Communications, 16(5), 2907-2920.

10. Hu, C., \& Hsu, C. (2019). Efficient adaptive subarrays in millimeter-wave MIMO systems with hybrid RF/baseband precoding/combining design. IEEE Systems Journal, 13(4), 3735-3746

11. Liu, F., Kan, X., Bai, X., Du, R., \& Zhang, Y. (2019). Two-stage hybrid precoding algorithm based on switch network for millimeter wave MIMO systems. Progress in Electromagnetics Research $M, 77,103-113$.

12. Saleh, A. A. M., \& Valenzuela, R. (1987). A statistical model for indoor multipath propagation. IEEE Journal on Selected Areas in Communications, 5(2), 128-137.

13. Telatar, E. (1999). Capacity of multi-antenna gaussian channels. European Transactions on Telecommunications, 10(6), 585-595.

14. Sahoo, S. K., \& Makur, A. (2013). Dictionary training for sparse representation as generalization of K-means clustering. IEEE Signal Processing Letters, 20(6), 587-590.

15. Liu, F., Bai, X., Shi, H., Du, R., \& Liu, H. (2020). Hybrid precoding with adaptive sub-connected architecture for mmWave massive MIMO systems. 2020 IEEE 31st Annual International Symposium on Personal, Indoor and Mobile Radio Communications, 1-6.

16. Gao, X., Dai, L., Han, S., I, C., \& Heath, R. W. (2016). Energy-efficient hybrid ana$\log$ and digital precoding for mmWave MIMO systems with large antenna arrays. IEEE Journal on Selected Areas in Communications, 34(4), 998-1009.

17. Yu, X., Zhang, J., \& Letaief, K. B. (2018). A Hardware-Efficient Analog Network Structure for Hybrid Precoding in Millimeter Wave Systems. IEEE Journal of Selected Topics in Signal Processing, 12(2), 282-297. 

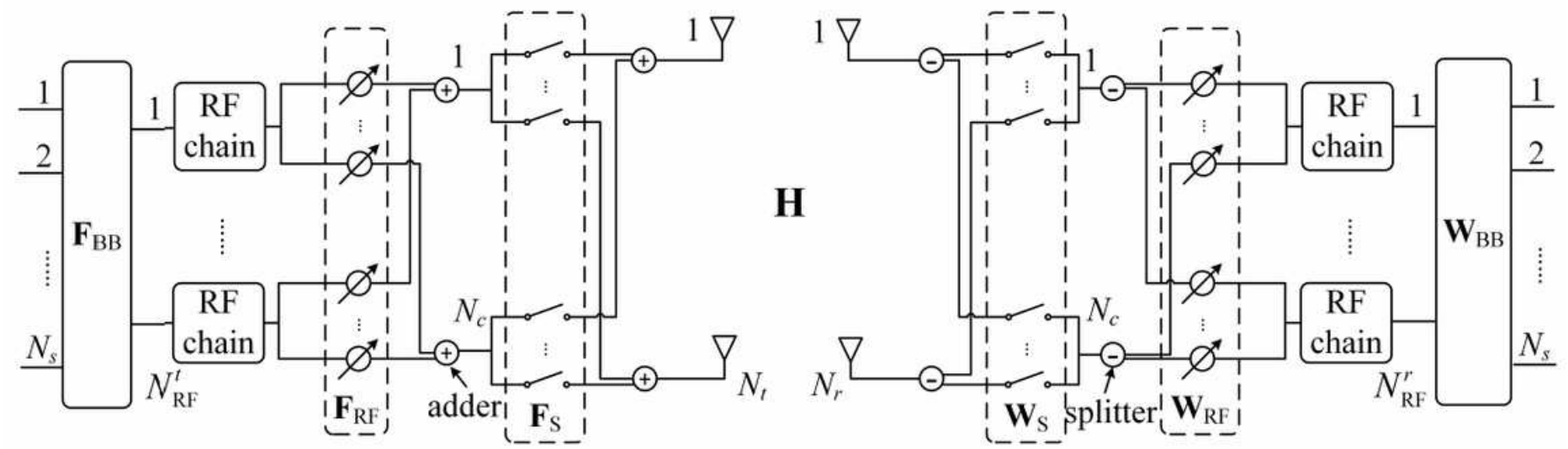

Figure 1

Single-user mmWave MIMO system based on the SFC structure.

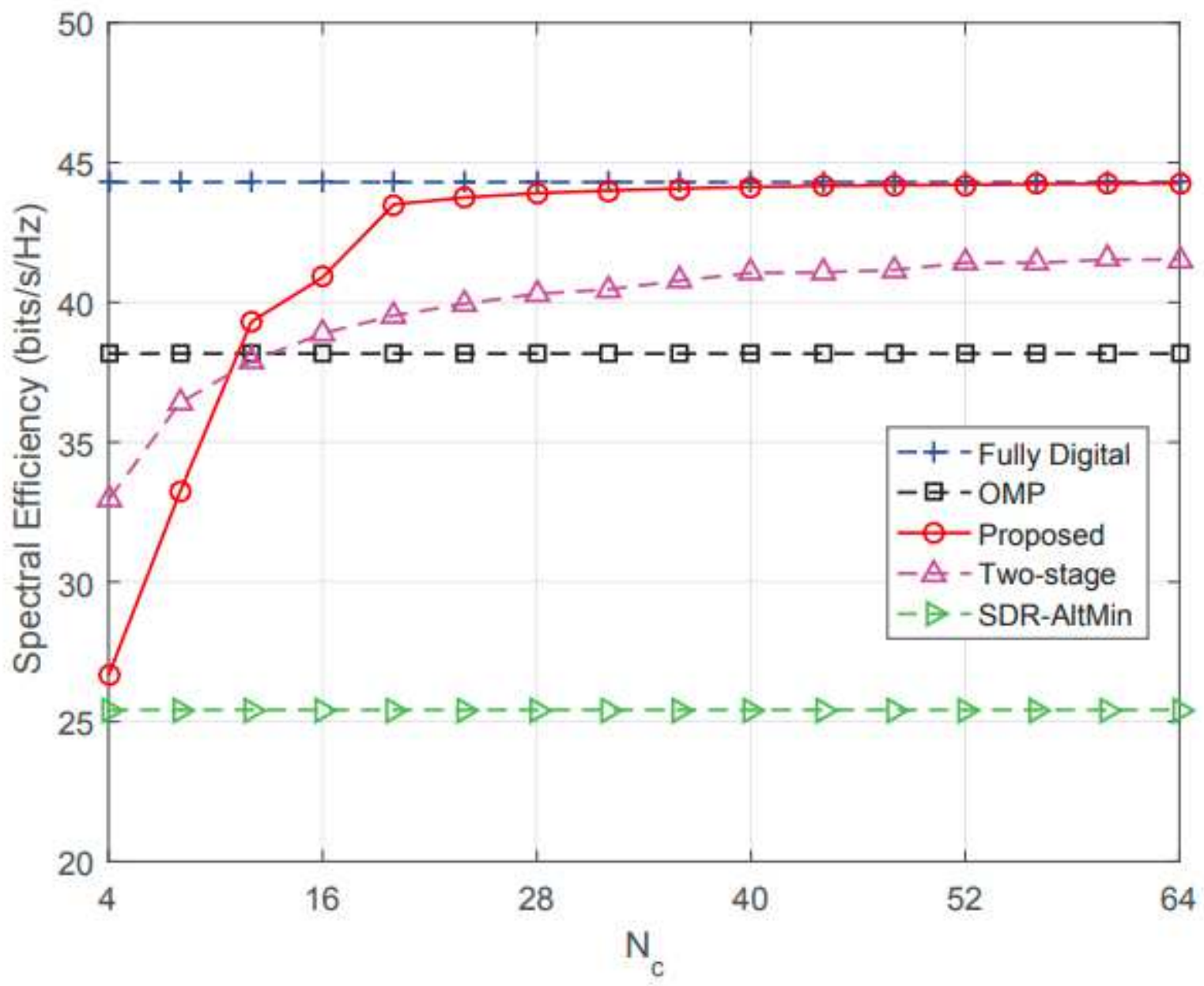

Figure 2 
Spectral efficiencies versus Nc.

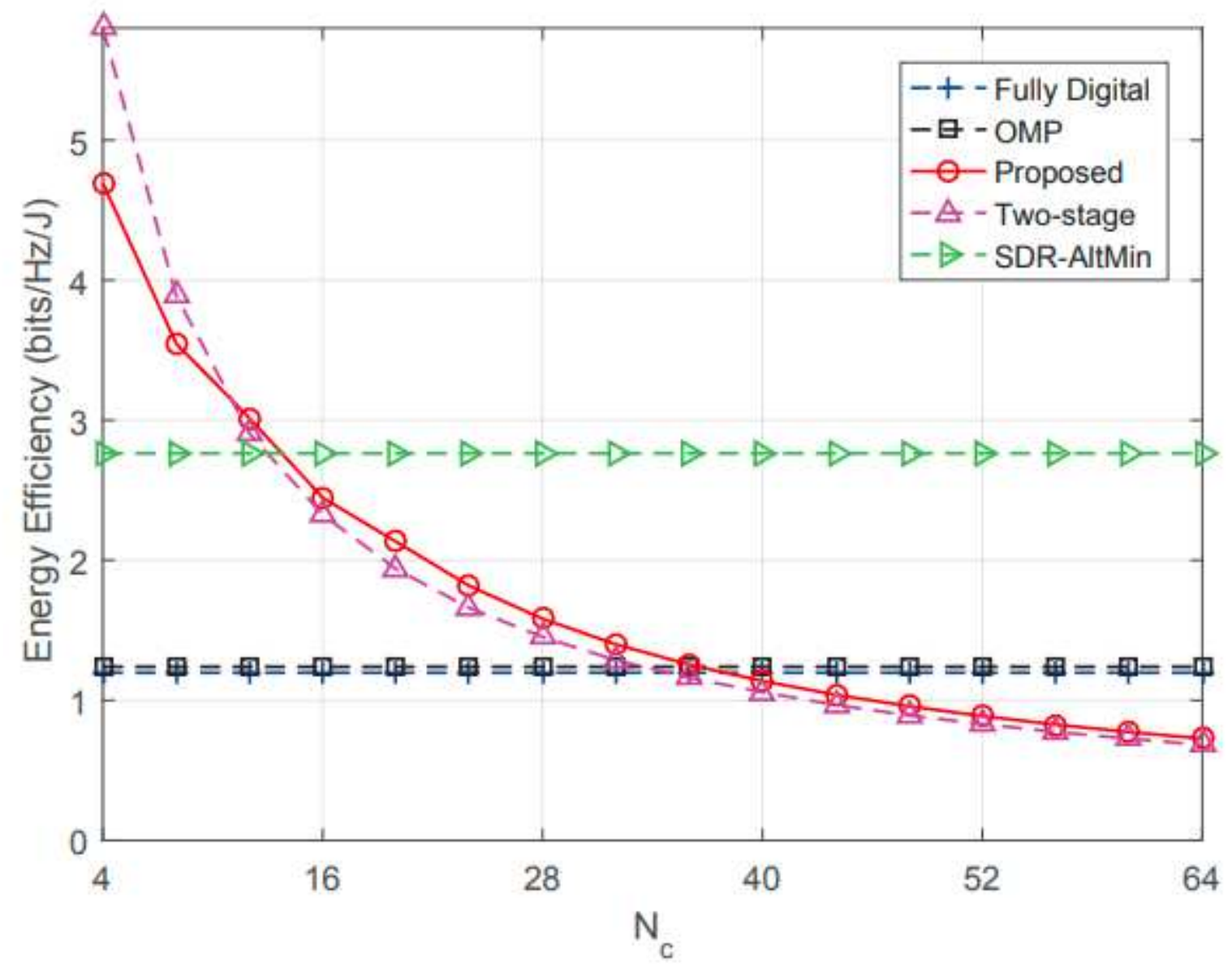

Figure 3

Energy efficiencies versus Nc. 


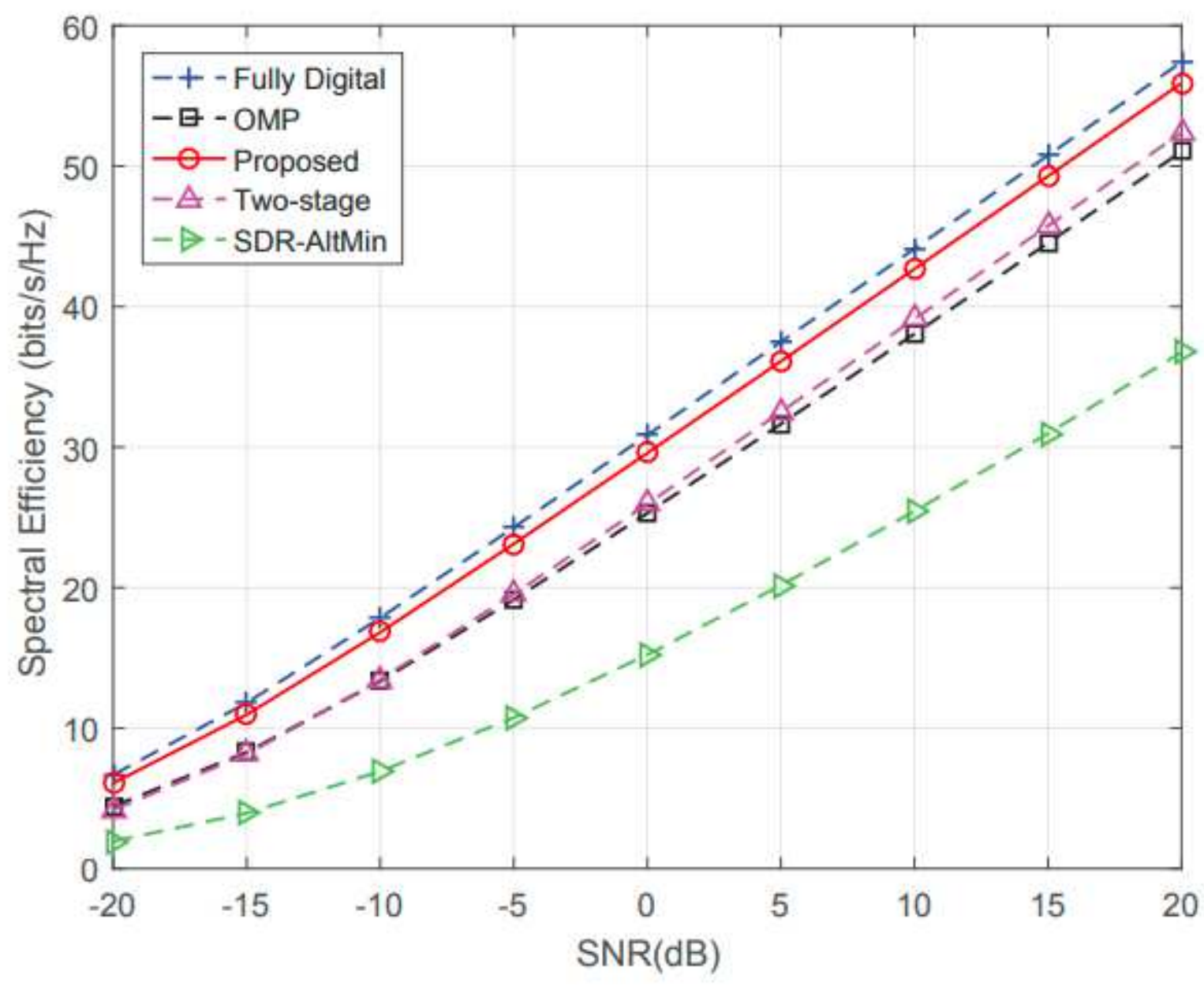

Figure 4

Spectral efficiencies versus SNR. 


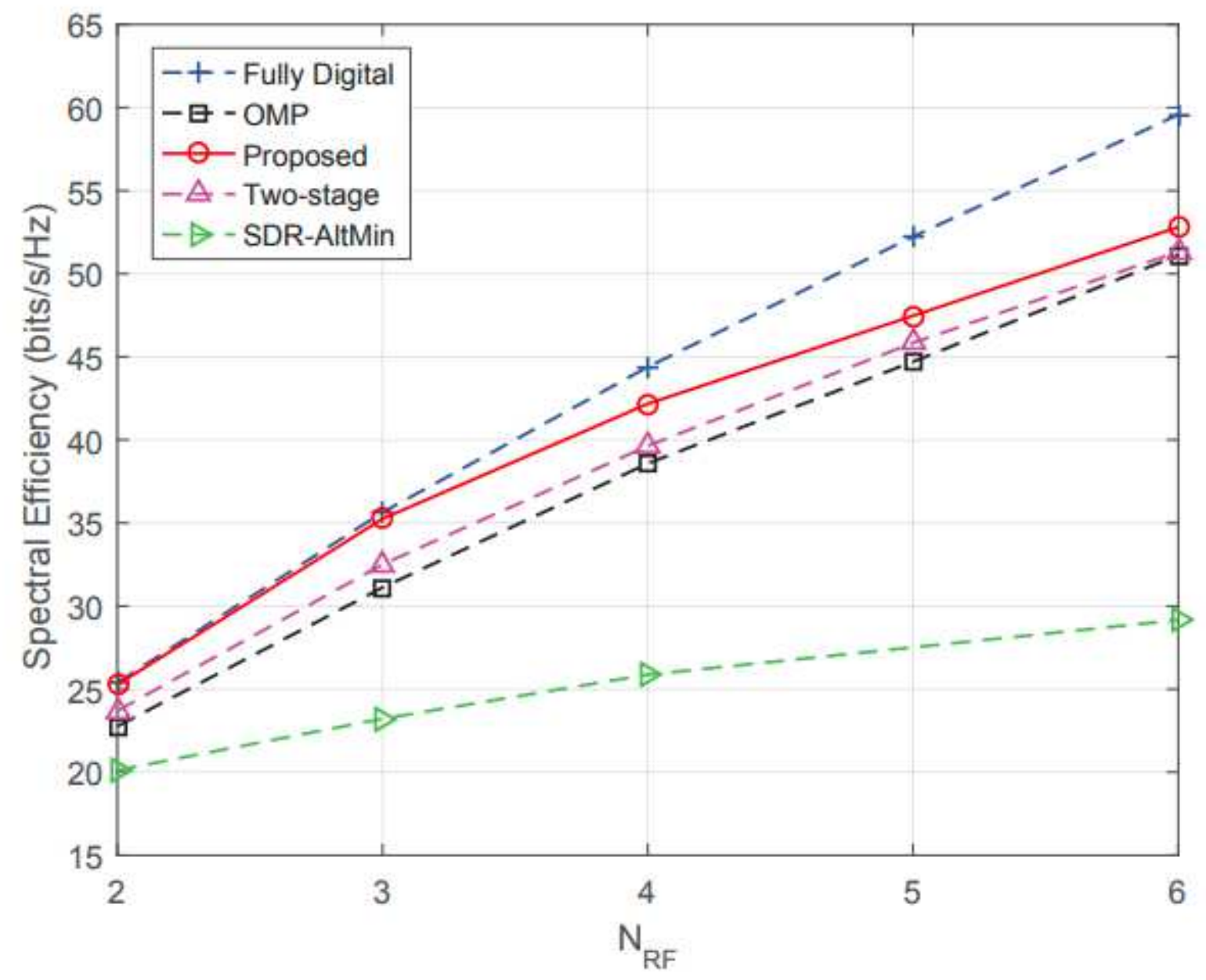

Figure 5

Spectral efficiencies versus NRF . 


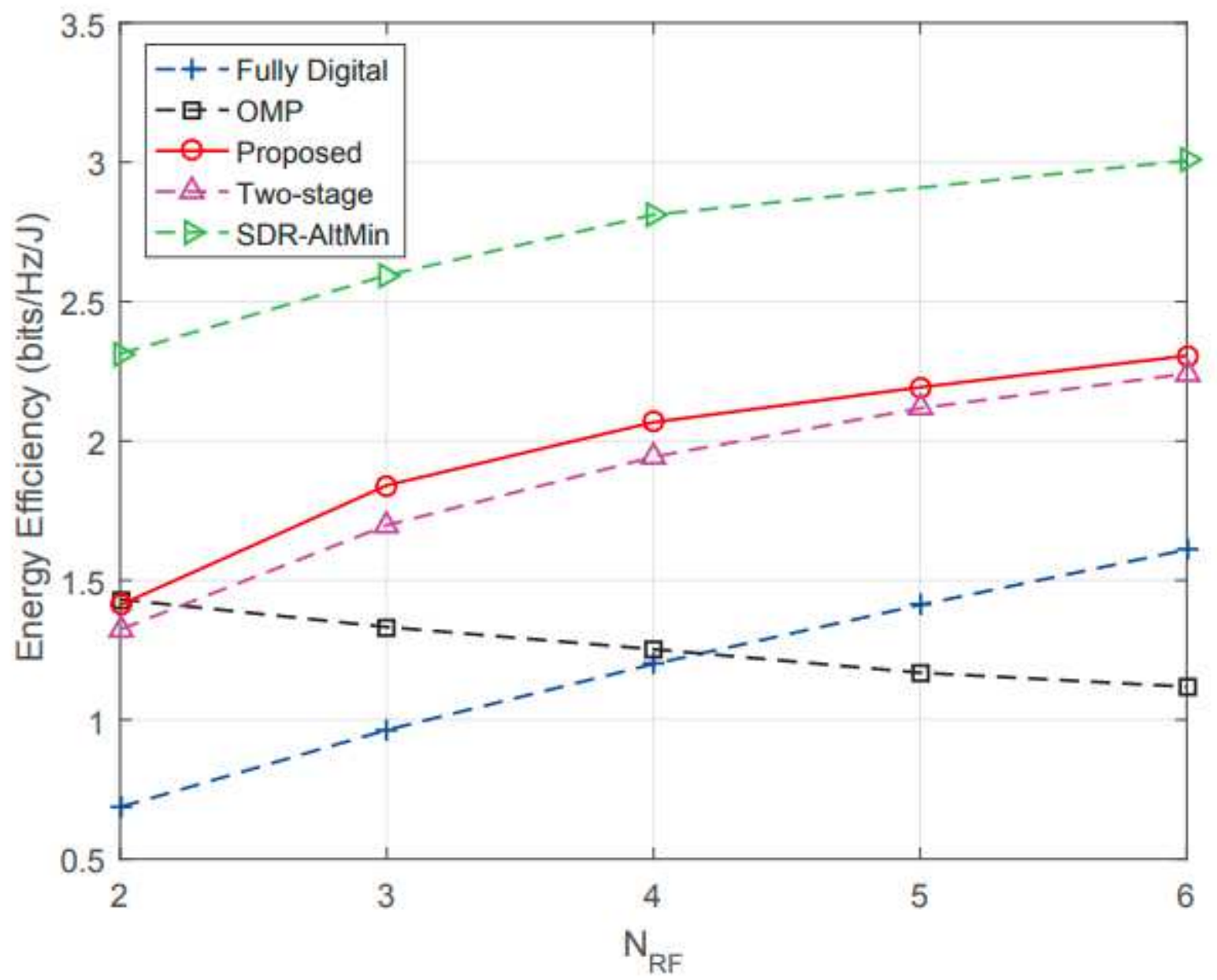

Figure 6

Energy efficiencies versus NRF . 


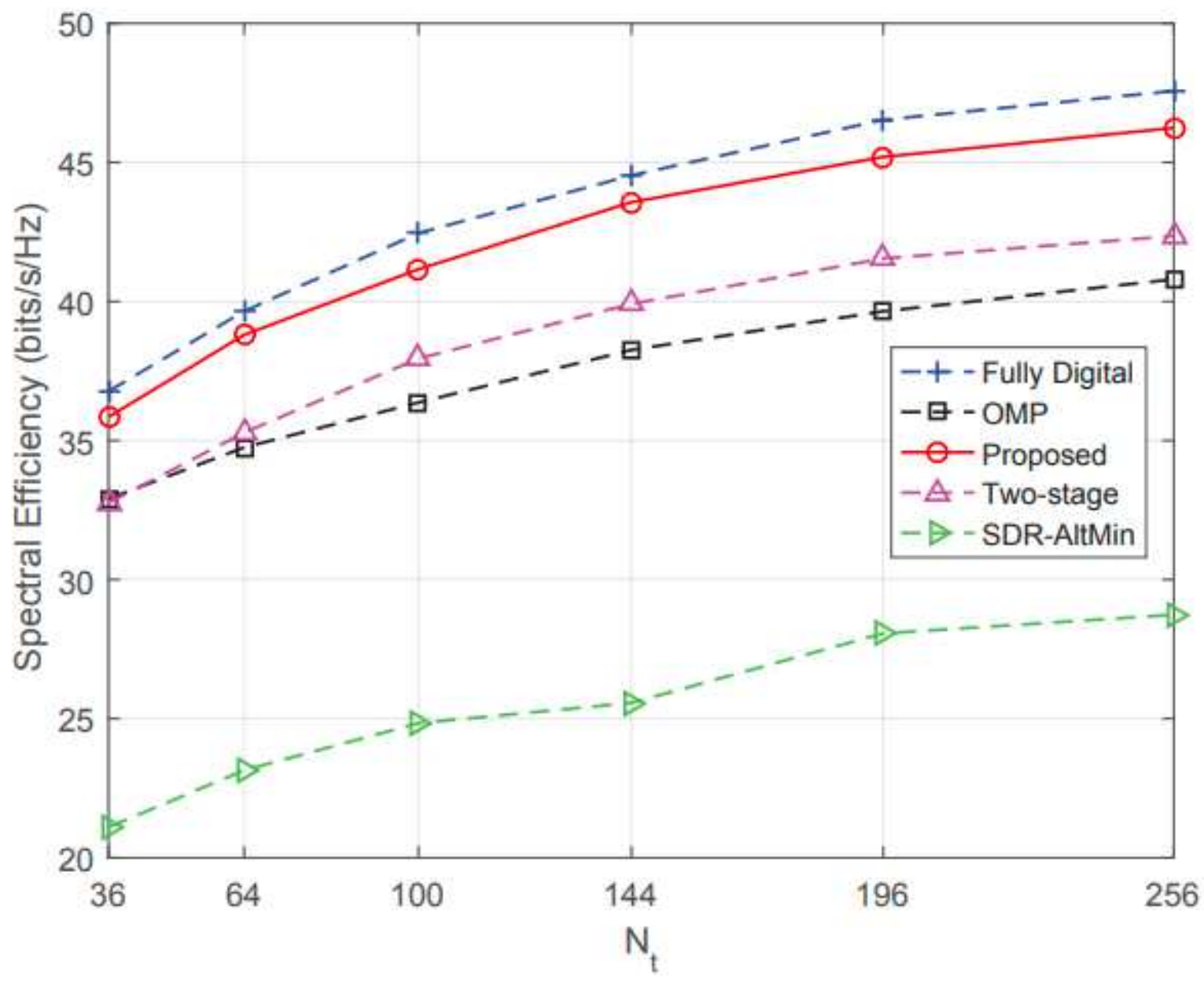

Figure 7

Spectral efficiencies versus Nt. 


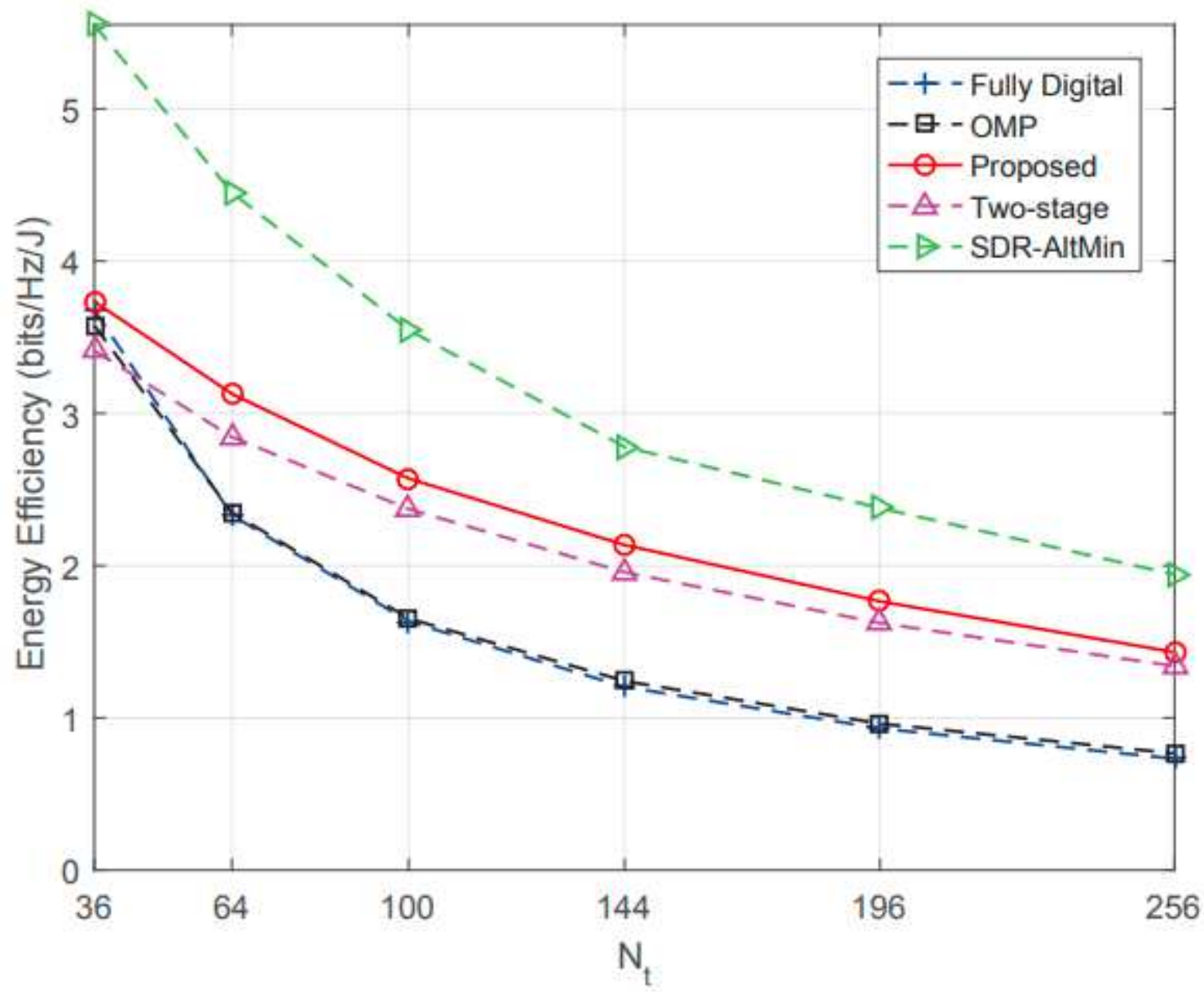

Figure 8

Energy efficiencies versus Nt. 